\title{
Un reto informativo: Hismedi, la transición española y la historia multimedia
}

\author{
An Informational Challenge: HISMEDI, Spanish Transition \\ and Multimedia History
}

\author{
Raúl Magallón Rosa \\ Universidad Carlos III de Madrid \\ https:// orcid.org/0000-0002-2236-7802 \\ raul.magallon@uc3m.es
}

Recibido: 12/09/2021 ; Revisado: 24/01/2022; Aceptado: 27-01-2022

\begin{abstract}
Resumen:
El objetivo de esta investigación es estudiar las nuevas formas de transmisión del pasado y, concretamente, las fuentes digitales de la transición española que configuran la nueva esfera pública digital. Para ello se describe y analiza la base de datos del proyecto Hismedi-Transición a la democracia, con el objetivo de distinguir y cuantificar las diferentes fuentes digitales que forman parte de sus colecciones. En la actualidad, los recursos pueden dividirse en: blogs, documentales en plataformas de vídeo, especiales de medios de comunicación, páginas webs, bases de datos, redes sociales como Twitter o Facebook, portales educativos, boletines y newsletters.
\end{abstract}

Palabras clave: España, transición, historia multimedia, fuentes digitales, memoria.

\begin{abstract}
:
This article looks at new ways of putting the past on display, specifically the digital sources pertaining to the Spanish transition that constitute the new digital, historical and journalistic public sphere. The article describes and analyses the database resulting from the HISMEDI: Political Transition to Democracy project, with the aim of distinguishing and quantifying the different digital sources that are part of its collections. Currently, resources can be divided into: blogs, documentaries on video platforms such as YouTube or Vimeo, media features, websites, databases, social networks such as Twitter or Facebook, educational portals, bulletins and newsletters, etc.
\end{abstract}

Keywords: Spain, Transition, Multimedia History, Digital Sources, Memory. 


\section{INTRODUCCIÓN ${ }^{1}$}

El objetivo de esta investigación es estudiar las nuevas formas de transmisión del pasado y, en concreto, las fuentes digitales de la transición española que configuran la nueva esfera pública digital, histórica y periodística. Para ello se describe y analiza la base de datos del proyecto Hismedi-Transición a la democracia, ${ }^{2}$ con el objetivo de distinguir y cuantificar las diferentes fuentes digitales que forman parte de sus colecciones.

A nivel global, las preguntas que el proyecto plantea son las siguientes: ¿Qué aspectos de la transición española despiertan mayor interés a los ciudadanos? ¿Coinciden con el interés historiográfico? ¿Cuál es la contribución de la sociedad digital en la generación del conocimiento sobre la etapa? ¿Qué papel tienen las redes sociales en la revisión del significado de la transición? ¿Quiénes son los autores y en qué fuentes se basan, teniendo en cuenta la cercanía cronológica de la fase? ¿Qué papel tienen los historiadores en la nueva transmisión de la ciencia histórica «con la sociedad»?.

Este trabajo no tiene como objetivo responder a las mismas de forma completa sino intentar explicar el marco de reflexión en el cuál se integra el proyecto. De este modo, resultaba fundamental establecer previamente una taxonomía con palabras clave sobre la Transición a la Democracia para facilitar las búsquedas, identificación, selección y descripción de las fuentes digitales objeto de estudio y construir una base de datos para incorporar los nuevos registros y entornos virtuales.

Como sabemos, en la actualidad, la transmisión de la historia y de la memoria de nuestro pasado ha dejado de ser monopolio de los historiadores y multitud de agentes sociales contribuyen con información y opinión a la configuración de conocimiento histórico. El trabajo del historiador queda redefinido por el lugar -a veces, secundario- que adquiere la cultura escrita en este nuevo escenario digital frente a una situación previa en la cual la propia estructura estática del documento impreso generaba una confianza (Pons, 2006).

Eiroa, en esta línea, subraya que tradicionalmente la historiografía general «ha aplicado técnicas metodológicas basadas en la síntesis histórica tradicional, o la ordenación cronológica de la información puesto que su interés ha residido en utilizar la información vertida en sus páginas como evidencias del acontecer social y político del pasado» (EIROA, 2019). Sin embargo, en la actualidad los recuerdos individuales y colectivos creados y tratados de forma digital son «una expresión mundial de la participación popular en la construcción de un pasado colectivo y, a la vez, son el producto de esos nuevos soportes digitales» (NOIRET, 2018).

Este ecosistema otorga a los historiadores una función de divulgación con distintos niveles comunicativos: la comunicación de la historia a un público no académico, la participación pública y la aplicación de la metodología histórica a las cuestiones actuales (PONS, 2020: 72; CAUVIN, 2018). De este modo, la historia ha entrado en el debate social y político del presente en respuesta al interés

1 Este trabajo se inscribe como resultado del proyecto de investigación Historia, memoria y sociedad digital. Nuevas formas de transmisión del pasado. La transición política a la democracia, financiado por el Programa Retos del Conocimiento, del Ministerio de Ciencia, Investigación y Universidades y el Fondo Europeo de Desarrollo Regional (FEDER) con referencia RTI2018-093599-B-I00 MCIU/AE/ FEDER, UE, MCIN/AEI/10.13039/501100011033/FEDER «Una manera de hacer Europa».

2 Página web de hismedi: https://humanidadesdigitales.uc3m.es/s/hismedi/page/inicio 
manifestado por los ciudadanos, pero también por nuevos agentes políticos y sociales que buscan recuperar determinados relatos del pasado como bandera de causas actuales.

Nos encontramos, por tanto, ante nuevos modelos de transmisión, nuevas narrativas, así como diversas significaciones y públicos que se enmarcan en el contexto de una memoria activa pero, en ocasiones, también transformadora del presente. La memoria, en este ecosistema, se presenta como un cruce entre el relato individual, los documentos públicos y las narraciones sociales, pero también como una estructuración de la complejidad de las sociedades actuales que permite la exploración y análisis de acciones futuras basadas en la reivindicación de un tipo de pasado (MAgAllón-Rosa, 2008). Una memoria que se basa precisamente en el desafío de elegir qué recordar en un nuevo ecosistema donde lo que existe, precisamente, es un exceso de información.

\section{MARCO TEÓRICO. ACONTECIMIENTOS HISTÓRICOS, MEMORIA PÚBLICA Y DOCUMENTOS AUDIOVISUALES}

Hasta ahora, el análisis que los medios de comunicación habían desarrollado sobre el pasado, se había realizado principalmente desde dos perspectivas: la de los historiadores en general -centrado principalmente en el análisis de acontecimientos y personalidades- y la de los historiadores de la comunicación, cuyo objeto de estudio eran los medios en sí mismos.

Sin embargo, y puesto que casi toda la Historia del futuro ya siempre quedará registrada y las fuentes -que durante tanto tiempo fueron escasas- llegarán a ser prácticamente ilimitadas, parece claro que la revisitación de los discursos de poder se centrarán no tanto en intentar representar la realidad -ya que en muchas ocasiones podremos a acudir a la fuente primaria para extraer nuestras propias conclusiones- como en reformular los errores e interpretaciones que ese pasado generó.

Fickers, al respecto, señala que «desde el principio, el trabajo histórico estuvo caracterizado por una doble ambición: primero, encontrar y recoger fuentes históricas como trazas de tiempos pasados (el historiador como archivero y cronista); en segundo lugar, producir una narrativa coherente del pasado, interpretando las fuentes basándose en preguntas e intereses contemporáneos (el historiador como intérprete)» (FICKERS, 2012).

En la actualidad, los acontecimientos mediáticos han de entenderse para siempre como monumentos electrónicos (digitales). Y en ellos, el discurso de la memoria -principalmente de la colectiva-, se establece como forma de consenso y jerarquización de diferentes valores, pero también como batalla cultural entre realidades enfrentadas.

Paralelamente, comprender los contextos y las prácticas analíticas en las que se insertan estos acontecimientos históricos ayudar a definir el objeto de estudio. En este sentido, Pons señala que «comprender la historia digital exige percibir el contexto en el que se produce y entender qué defienden sus practicantes, quienes, de un modo $u$ otro, asumen que el medio es el mensaje o que, al menos, forma parte de él» (PONS, 2018: 22).

Por su parte, EGIDo y EIROA (2017) nos recuerdan que las nuevas fuentes transmedia nos remiten al conocimiento de un pasado distinto y esto supone un 
reto para los historiadores en el que se distinguen:

1.- Las fuentes digitales que pueden contribuir a la construcción histórica de los acontecimientos.

2.- Un conjunto de aportaciones bien documentadas, obra de colectivos que muestran la historia de los olvidados/silenciados y reivindican su sitio en la historia.

3.- La contribución oral de testigos que prestan su testimonio sobre su experiencia vital.

En concreto, y en relación a la transición, aparece el problema de las fuentes documentales olvidadas, de las no publicadas, de los silencios todavía vigentes y, por qué no decirlo, de las fuentes por descubrir. Como subraya Cuesta (2007), «el problema del silencio o del olvido en la transición aflora permanentemente. Paradojas de la memoria que se pliega a circunstancias políticas, olvidando realidades que un día fueron palmarias y, lo que es más grave, paralizando sus consecuencias y congelando derechos».

\subsection{La evolución de las fuentes digitales: usos sociales e interpretaciones}

Las características de internet y la posibilidad de incluir elementos multimedia -fotografías, voz, sonidos, documentos textuales-, hipertextos o la interacción, habilitan la multiplicación del conocimiento, pero sobre todo promueven la segmentación de relatos del presente (EGIDO y EIROA, 2017). Mención especial, por su novedad, son los boletines y las newsletters históricas que constituyen un conjunto de fuentes primarias de gran valor para la historiografía y «que contribuyen a una visión más social, más emotiva y cercana de los eventos y personajes que retratan» (EIROA, 2020:110).

Estos relatos necesitan también de nuevos conocimientos digitales que permitan integrar esa segmentación y adaptar los tiempos de la investigación histórica. Como apunta Noiret, «comprender en la actualidad cómo se utiliza la fotografía en las redes sociales y cómo participa en la historia dice mucho sobre qué pasados son los que importan en nuestro presente» (NOIRET, 2018).

Sin embargo, cuando empezamos a asociar la Historia con la cultura digital, nos encontramos con que los tradicionales criterios cronológicos (por ejemplo, la historia de la Edad Media, la historia contemporánea, etc.) o temáticos (historia urbana, historia económica, etc.) «siguen siendo preferidos a categorías Web 2.0» (GALLINI y NOIRET, 2011).

EGIDO y EIROA (2017), en esta línea, señalan que «una cuestión clave a la hora de abordar las fuentes digitales es comprobar su calidad y credibilidad, una evaluación obligada no sólo para usos historiográficos sino también para la consulta como internautas».

Las nuevas fuentes digitales conviven con la demanda de una cultura de la transparencia en la que el papel de los datos abiertos y de la información pública se puede plantear desde dos perspectivas: por un lado, desde aquellos acontecimientos del pasado que se reactualizan por la aparición de nueva fuentes que amplían, añaden o modifican el conocimiento sobre el suceso y, por otro lado, desde el presente con aquellos hechos noticiosos que desde el momento en el que se producen marcan el desarrollo de la historia futura (MAGALLón-RosA, 2017).

En la actualidad, la historia del futuro no sólo será contada a través de 
archivos históricos y fuentes periodísticas. Cada vez es más frecuente pensar en los productos de ficción como mecanismos de transmisión cultural e histórica. En este sentido, hay que recordar que los archivos audiovisuales «cumplen una doble misión cultural. En primer lugar, una misión histórico-educativa, servir como testigo del pasado reflejando historias, vidas, costumbres o creencias, por citar sólo algunas. Y, además, una misión transmisora conservadora encargada de seleccionar qué se preserva para generaciones futuras y cómo debe hacerse para asegurarse de que el material transcienda a los tiempos» (RODRíGUEZ y HERNÁNDEZ, 2015).

\subsection{Fuentes documentales. La historia multimedia de la transición española}

La abundancia de fuentes audiovisuales y mediáticas en torno a la transición y la proximidad de esta fase histórica con acontecimientos de actualidad han generado un importante conjunto de fuentes digitales que es necesario analizar, filtrar y seleccionar. Las efemérides, y el desarrollo de las redes sociales, han rescatado historias personales que se convierten en elementos continuadores de la memoria colectiva y social de la transición.

Junto a la figura de Juan Carlos I emerge la de Adolfo Suárez a la hora de describir los dos pilares fundamentales de la transición. Sin embargo, el halo de opacidad y secretos que todavía permanece sobre este periodo alimentan la aparición constante de este tipo de fuentes documentales «alternativas». De hecho, y teniendo en cuenta que en España la Ley de Secretos Oficiales data de 1968, queda aún mucha documentación escrita -y quién sabe si audiovisual- por descatalogar.

Paradójicamente, esta opacidad institucional, ha hecho que el relato oficial de la transición esté siendo transformado por acontecimientos recientes que relacionan a sus protagonistas -principalmente la figura de Juan Carlos I- y no por los propios sucesos que determinaron aquel periodo histórico. Como sabemos, el papel de la prensa como actor fundamental a la hora de moldear los consensos de la transición está siendo revisitado por los propios silencios que ésta permitió u ocultó.

Al respecto, tal y como recuerda Zugasti, «durante el período en que institucionalmente se completó la transición, los periódicos forjaron la imagen de Juan Carlos I que se ha mantenido hasta nuestros días, caracterizada fundamentalmente por el énfasis puesto en su papel como actor democratizador» (ZugASTI, 2005). En la misma línea, Carratalá defiende que «el discurso hegemónico, producido y difundido por los medios, ha otorgado una cobertura privilegiada a la institución. Mediante ese pacto tácito de complicidad, prensa, radio y televisión se han sometido a un tratamiento que combinaba dosis de amnesia con otras de tolerancia, ignorancia y aproximación benévola a aquellos aspectos más sensibles vinculados con la Casa Real» (CARRATALÁ, 2015).

En cualquier caso, y a medida que los secretos oficiales han permanecido, se ha abierto una polémica académica, social y política en cuanto a la interpretación y revisión del discurso oficial ligado al consenso y a los acuerdos alcanzados por los distintos actores sociales durante la transición. 


\section{UN ANÁLISIS DEL PROYECTO HISMEDI. METODOLOGÍA Y RESULTADOS}

El proyecto HISMEDI -Historia, Memoria y Sociedad Digital. Nuevas formas de transmisión del pasado. La transición política a la democracia- se presenta como un recurso audiovisual de catalogación de fuentes digitales para investigar determinadas problemáticas relacionadas con la transición española. El proyecto se inició en 2019 y la actualización no ha seguido una periodicidad concreta.

Para su creación se elaboró una taxonomía con conceptos y personajes de la transición y con los tesauros de PARES, del archivo Linz y del proyecto TRANSLITEME. Con estos conceptos se procedió a la búsqueda de fuentes disponibles y los hallazgos se categorizaron según los metadatos recogidos por Dublin Core para ser incluidos posteriormente en el software de OMEKA -que funciona como una base de datos-.

\section{HISMEDI - TRANSICIÓN A LA DEMOCRACIA}

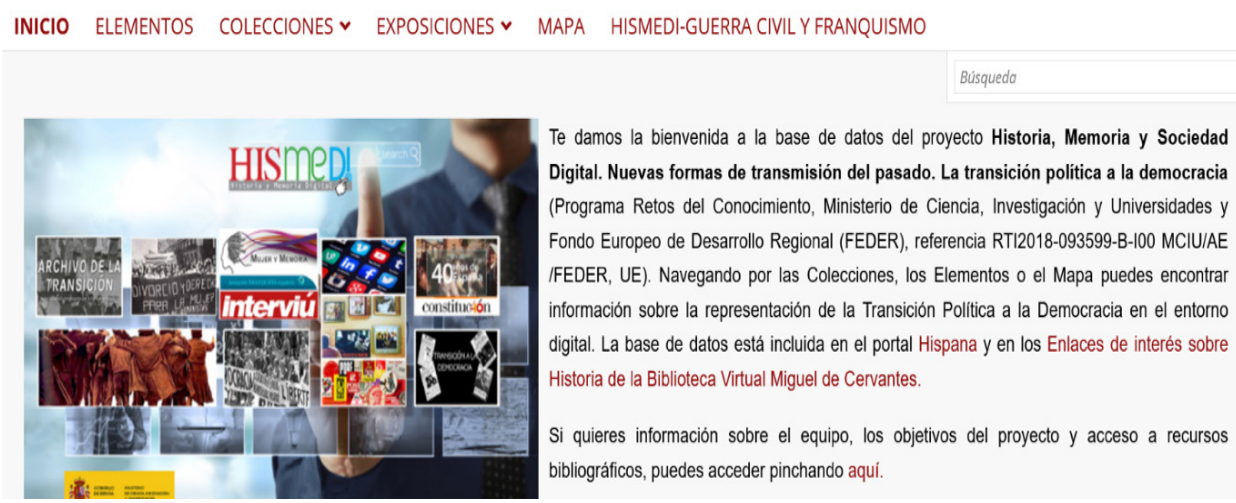

Figura 1. Proyecto HISMEDI-Transición a la Democracia.

Fuente: Página web del proyecto.

Con los metadatos se procedió al análisis de los autores, el tipo de registro, la fecha de creación, el tema, etc. Es decir, se tomó el proceso de describir, identificar y procesar previamente para poder analizar e interpretar posteriormente. Por otra parte, es necesario señalar que el software de OMEKA no permite la cuantificación de la audiencia. Sin embargo, Hismedi-Transición, al igual que Hismedi-Guerra Civil y Franquismo, ha sido recogido por el portal HISPANA que reporta a la biblioteca digital Europeana y, por lo tanto, se puede considerar que -potencialmente- tiene una amplia audiencia de docentes e investigadores. También figuran entre los enlaces de interés sobre Historia que recoge la Biblioteca Virtual Miguel de Cervantes, así como en algunos blogs y webs de referencia en el campo de estudio.

En este momento el proyecto establece como criterios de entrada, la selección 
de «formatos» o «géneros» de internet para clasificar los hallazgos. Es decir, tiene como categorías la división en blogs, las webs, videos, etc. Esta organización por formatos es homologable a la que se hace en los archivos tradicionales, donde clasifican la documentación por fechas pero también por «géneros», por ejemplo: Informes, Expedientes personales, Correspondencia, Imágenes, etc.

A medida que se incorporan más elementos clasificados se puede realizar una organización temática, como ya se hizo en las exposiciones virtuales de Hismedi-Guerra Civil y Franquismo. ${ }^{3}$ De esta forma se podrían realizar exposiciones virtuales de, por ejemplo, La Constitución, La violencia en la transición (La matanza de Atocha, por ejemplo), los partidos políticos, Adolfo Suárez, etc.

Esta tematización ha permitido que en la actualidad las exposiciones versen sobre Mujeres en el ecosistema digital de la transición a la democracia en España: recursos para su estudio y El PCE y la izquierda revolucionaria en la transición a la democracia: recursos para su estudio. ${ }^{4}$

La exposición sobre mujeres en la transición se centra en los siguientes aspectos: Archivos, bibliotecas y repositorios; Feminismo y Activismo feminista; Proyectos de investigación; Historia de las mujeres; Mujeres sobresalientes y Violencia política. Por otra parte, Archivos y Documentos, Asesinatos de Atocha, Legalización del PCE, Activismo político, Actividad sindical, Izquierda revolucionaria y Protagonistas integran la exposición sobre el PCE.

En este contexto de catalogación, los recursos que aparecen en las colecciones pueden dividirse en: blogs, plataformas de vídeo como Youtube o Vimeo, especiales de medios de comunicación, páginas webs, bases de datos, redes sociales como Twitter o Facebook, portales educativos, etc.

$\mathrm{Al}$ respecto, y desde una perspectiva digital, es importante señalar que en los archivos audiovisuales aparece la necesidad de valorar la importancia del código y de la estructura abierta (CoHEn y RosenzweIG, 2005). Como recuerda Eiroa, «cada medio tiene su propia historia y convenciones y es necesario ser consciente de que la información historiográfica depende del tipo de información primaria que haya permanecido» (EIROA, 2018: 88).

Para cumplir con esta premisa, el proyecto recoge las siguientes colecciones:

- Redes sociales: Instagram, Twitter y Facebook.

- La Webesfera de la Transición.

- La Blogosfera de la Transición.

- Documentales y videos en Youtube.

- Reportajes especiales en medios de comunicación.

- Boletines y Newsletters.

En este sentido -y antes de describir los resultados obtenidos en profundidades necesario tener en consideración que las propuestas para el análisis, la verificación de la calidad y credibilidad de los recursos digitales de HISMEDI pretende -siempre que ha sido posible- incluir «indicadores de identidad como el autor/entidad emisora, la fecha de creación, la estructura y organización interna, la financiación o soporte económico y una explicación de los objetivos» (EGIDO y EIROA, 2017). Esta idea coincide con la de Noiret que señala que un «archivo material» útil empieza teniendo en cuanta la historia de la producción documental

3 Hismedi-franquismo. Disponible en: https://uc3m.libguides.com/hismedi_franquismo 4 Véase: https://humanidadesdigitales.uc3m.es/s/hismedi/page/exposiciones 
y las normas y criterios con los que se ha creado éste.

\subsection{Resultados}

Las palabras clave, junto con las colecciones, son las dos formas de categorización que tiene la base de datos de Hismedi de la transición. Al respecto, un análisis de la base de datos y sus palabras clave nos permite confirmar que los términos violencia, historia general, política y protagonistas son los más frecuentes a la hora de categorizar las fuentes digitales, incorporar nuevos registros y seleccionar el tipo de fuente.

Puesto que hablamos de la transición y un tipo de sociedad emergente que se va configurando en torno a las nuevas libertades, también destaca la aparición de nuevos movimientos sociales y políticos como forma de categorizar y organizar la información: feminismo, terrorismo o movimiento obrero son los más frecuentes.

Como señalábamos, la base de datos que recoge el proyecto HISMEDI divide sus colecciones en: redes sociales (Twitter, Facebook Instagram, etc.), la Webesfera de la Transición, la Blogosfera de la Transición, documentales y videos en Youtube, reportajes especiales en medios de comunicación y boletines y Newsletters.

Dentro de las distintas colecciones, las temáticas pueden variar por: colectivos, lugares con una fuerte connotación histórica, acontecimientos históricos, discursos, personajes, testimonios orales, archivos, etc. Hasta la fecha recoge un total de 652 entradas que permiten establecer un primer análisis en torno a temas, plataformas o canales y palabras clave. ${ }^{5}$

Distribución de palabras clave de la base de datos HISMEDI

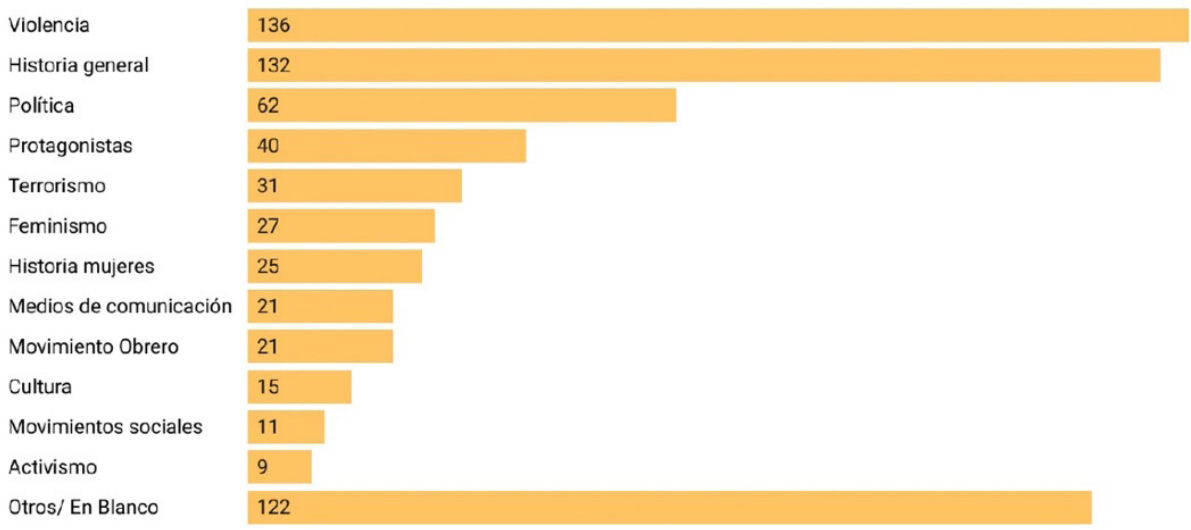

Fuente: Hismedi. Elaboración propia.

5 Septiembre de 2021. 
GRÁFICO 2

Distribución del tipo de fuente digital de las colecciones

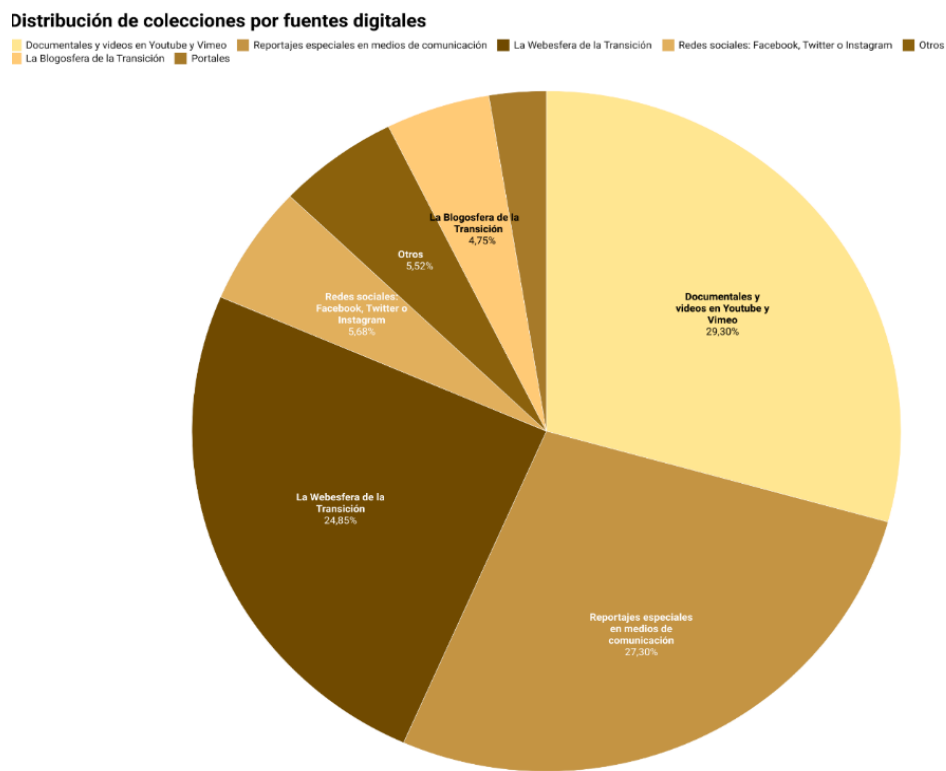

Fuente: Hismedi. Elaboración propia.

De los recursos digitales catalogados, debemos señalar que la blogosfera es quizá la más limitada, en cuanto que se trata principalmente de entradas concretas sobre determinados temas o acontecimientos. El reducido número de blogs que se encuentran se debe a su estructura y al tipo de «medio». Es decir, a que se configuran a modo de nuevos cuadernos virtuales donde expresar la opinión personal, pero también como homenaje a acontecimientos próximos, memoria de familiares, documentos procedentes de archivos privados, etc.

Una posible hipótesis es que los protagonistas de la transición y sus herederos no utilizan con la misma frecuencia los blogs como forma de activación de la memoria, no utilizan este formato para escribir sus memorias personales. Este es un dato interesante en contraposición con la colección de blogs de guerra civil y franquismo, donde este tipo de formatos son muy abundantes (EIROA, 2019).

En relación al franquismo, los protagonistas $\mathrm{y}$, sobre todo, sus herederos (nietos, asociaciones, etc.) habían decidido volcar en un blog su memoria personal, archivos privados con fotos, cartas, etc., en muchos casos con un tono reivindicativo de la actuación que realizaron ante esos hechos dramáticos. Sin embargo, y como hemos podido comprobar, no ocurre así en la transición. 
Un reto informativo: Hismedi, la transición española y la historia multimedia

TABLA 1

Distribución porcentual del número de entradas de cada colección

\begin{tabular}{|c|c|c|c|}
\hline Colección & $\begin{array}{l}\text { Número de } \\
\text { entradas }\end{array}$ & Porcentaje & Temas \\
\hline $\begin{array}{l}\text { Redes sociales: } \\
\text { Facebook, Twitter o } \\
\text { Instagram }\end{array}$ & 37 & $5,67 \%$ & $\begin{array}{l}\text { - Aniversario de la constitución. } \\
\text { - Archivos de la transición. } \\
\text { - Militancia femenina. } \\
\text { - Bebes robados. } \\
\text { - Transición y prensa. }\end{array}$ \\
\hline $\begin{array}{l}\text { La Webesfera de la } \\
\text { Transición }\end{array}$ & 162 & $24,84 \%$ & $\begin{array}{l}\text {-Colecciones fotográficas de acontecimientos y } \\
\text { personajes de la vida cotidiana. } \\
\text {-40 años de periodismo en democracia. La } \\
\text { información parlamentaria de la transición. } \\
\text { - Personajes clave: Adolfo Suarez. } \\
\text { - Archivos de sindicatos, fundaciones, lugares, } \\
\text { etc. } \\
\text { Archivo de temáticas: ecologismo, feminismo, } \\
\text { víctimas del terrorismo, movimiento LGTBI, } \\
\text { Etc. }\end{array}$ \\
\hline $\begin{array}{l}\text { La Blogosfera de la } \\
\text { Transición }\end{array}$ & 31 & $4,75 \%$ & $\begin{array}{l}\text { - Historia general. } \\
\text { - Presos del franquismo. } \\
\text { - Feminismo y transición. } \\
\text { - Memoria de la transición. } \\
\text { - Cine en la transición. }\end{array}$ \\
\hline $\begin{array}{ll}\text { Documentales } & y \\
\text { videos en Youtube } & y \\
\text { Vimeo } & \end{array}$ & 191 & $29,29 \%$ & $\begin{array}{l}\text { - Historia: general, de mujeres, partidos } \\
\text { políticos, organizaciones, etc. } \\
\text { Movimientos: vecinal, obrero, cultural, etc. } \\
\text { - Acontecimientos: históricos, violentos, etc. } \\
\text { - Protagonistas. } \\
\text { - Cultura y cine. }\end{array}$ \\
\hline Portales & 17 & $2,6 \%$ & $\begin{array}{l}\text { - Congreso de los Diputados. } \\
\text { - Archivos. } \\
\text { - La Moncloa. }\end{array}$ \\
\hline
\end{tabular}




\begin{tabular}{|l|l|l|l|}
\hline $\begin{array}{l}\text { Reportajes y especiales } \\
\text { en medios de } \\
\text { comunicación }\end{array}$ & $\mathbf{1 7 8}$ & $27,3 \%$ & $\begin{array}{l}\text { - Historia general. } \\
- \text { Papel de los medios; El País con la } \\
\text { constitución, } \\
\text { - Acontecimientos: pactos de la Moncloa, 23- } \\
\text { F, legalización PCE, últimos fusilamientos } \\
\text { franquistas-. } \\
\text { Temáticas: terrorismo, cine y cultura. }\end{array}$ \\
\hline
\end{tabular}

Fuente: Elaboración propia a partir de datos de HISMEDI.

Por el contrario, el papel de los documentales y de los vídeos en plataformas como Youtube emerge como la principal categoría en lo que hace referencia al formato o canal de difusión. Casi una de cada tres fuentes digitales localizadas en estas plataformas tiene este formato audiovisual. La aparición de tecnologías de grabación y registro audiovisual y su vinculación individual y testimonial al recuerdo del contexto histórico permiten entender esta prevalencia de relatos personales y colectivos que se presentan de forma paralela al relato oficial de la época. Paralelamente, destacan los reportajes y especiales en medios que suponen más del 27 \% del total de fuentes digitales categorizadas. De hecho, en el análisis se aprecia una evolución en esta categoría con el desarrollo del proyecto como consecuencia de la cobertura de RTVE, la celebración de efemérides -como los 40 años- y un aumento del interés por la memoria de la transición. Entre reportajes especiales en medios, documentales en Youtube y la webesfera suman más del $80 \%$ del total de entradas en la base de datos.

Como mencionábamos, este porcentaje resulta lógico por el papel que desempeñó la TVE en la transición. También porque existe una ventaja técnica importante al haber podido guardar sus archivos y ponerlos a disposición pública. Por último, hay que subrayar que poco a poco se aprecia un mayor interés en medios regionales y autonómicos por cubrir determinados aspectos locales que ocurrieron durante la transición. La diversidad de fuentes personales y geográficas permite poco a poco localizar los distintos relatos personales y colectivos que jalonaron su memoria social. Sobre todo, de aquellos relatos vinculados a determinadas formas de violencia de la época.

\section{CONCLUSIONES}

Como hemos visto, los nuevos espacios de construcción historiográfica y memorial aluden a una sociedad más activa e implicada en la transmisión del pasado. En esta esfera, se establecen canales abiertos al gran público en los que la representación del pasado en los medios de comunicación es un tema que no puede faltar cuando se analizan las funciones políticas, sociales y culturales de la divulgación de la historia.

En el caso concreto de estudio, encontramos una historia secreta y silenciada en la transición -la de los secretos oficiales- y una historia multimedia donde cada vez convergen con más fuerza el relato mediático de los acontecimientos y las 
narrativas sociales de las fuentes digitales.

De este modo, al valor documental de la cronología y las etapas históricas, la cobertura de los acontecimientos históricos, personajes relevantes, etc. se une el relato autobiográfico -completamente fragmentado y por explorar- que nos ayudará a entender mejor los matices de una de las etapas más importantes de la historia de España.

Intentando responder a la pregunta de investigación «¿cuál es el aporte neto de esta investigación al corpus historiográfico sobre la transición española?», hemos de señalar que todavía no se puede evaluar el aporte neto al corpus historiográfico sobre la transición porque todavía se están analizando y explotando los datos que se han recopilado.

Como hemos señalado, parte de estos objetos se han organizado en dos exposiciones temáticas: una sobre mujeres en la Transición y otra sobre el PCE y la izquierda revolucionaria. Estos objetos y su organización en exposiciones aportan, por un lado, fuentes históricas digitalizadas de archivos, entidades oficiales y privadas y fuentes digitales nativas que contienen elementos multimediáticos muy relevantes para conocer el periodo con evidencias que completan y complementan a las convencionales.

Por otro lado, el proyecto está revelando aspectos de la transición muy interesantes, destacando el caso de la violencia documentada de determinados movimientos políticos e ideológicos en los diversos territorios y regiones de nuestro país. Igualmente está mostrando cómo el discurso institucional de la transición sigue siendo el predominante desde un punto de vista historiográfico. Es decir, gran parte de los documentos recolectados se refieren al papel de las elites políticas y específicamente al de Adolfo Suárez y Juan Carlos I.

Como ocurre con el caso de los medios de comunicación digitales, quién está detrás de un proyecto, desde cuándo, la periodicidad de la publicación o la comunidad que le siguen son algunos elementos -objetivos y subjetivos- que forman parte del nuevo contrato de credibilidad de las fuentes históricas digitales. En este sentido, debemos replantearnos -pensando en los historiadores del futurocómo serán las bases de datos del presente porque es evidente que se convertirán más pronto que tarde en una herramienta de la historia multimedia del futuro.

\section{REFERENCIAS}

Carratalá, A. (2015): «El tratamiento de la Monarquía española en las viñetas de los medios digitales». Revista Dígitos, 1: 127-154. http:/ /dx.doi.org/10.7203/ rd.v0i1.7

Cauvin, T. (2018): «The Rise of Public History: An International Perspective», Historia Crítica, 68: 3-26 https:/ / doi.org/10.7440/histcrit68.2018.01

Cuesta, J. (2007): «Las capas de la memoria: contemporaneidad, sucesión y transmisión generacionales en España (1931-2006)». Hispania Nova, 7.

CoHEN, D. (2010): «Is Google Good for History? ». Dan Cohen’s Digital Humanities Blog. www.dancohen.org/2010/01/07/is-google-good-for-history_

COHEN, D.; RosenZWEIG, R. (2005): «Digital history. A guide to gathering, preserving, and presenting the past on the web». Center for History and New Media. http:/ / chnm.gmu.edu/digitalhistory

EgIDO, A.; EIROA, M. (2017): «Redes sociales, historia y memoria digital de la 
represión de mujeres en el franquismo». Revista de Historiografía, 27 (2): 341361. https:/ / doi.org/10.20318/revhisto.2017.3977

EIroA, M. (2020): «Revistas y Newsletters digitales de Memoria: una comunicación en Red sobre la actualidad del pasado», ESTUDIOS SOBRE EL MENSAJE PERIOdístico, 26 (1): 103-112. https:/ / dx.doi.org/10.5209/esmp.67290.

EIROA, M. (2019): «Primary sources for a digital-born history: the Hispanic blogosphere on the Spanish Civil War and Franco's regime», Culture $\mathcal{E}$ History Digital Journal, 7 (2). https:/ / doi.org/10.3989/chdj.2018.016

EIROA, M. (2018): «El pasado en el presente: el conocimiento historiográfico en las fuentes digitales», Ayer, 110 (2): 83-109.

FicKers, A. (2012): «Towards a New Digital Historicism? Doing History In The Age Of Abundance», Journal of European Television History and Culture, 1 (1): 19-26. http:/ / doi.org/10.18146/2213-0969.2012.jethc004

Gallini, S.; NoIret, S. (2011): «La historia digital en la era del Web 2.0». Historia digital. Historia Crítica, 43: 16-37.

LEMUS, E. (2008): «Los Estados Unidos y la imagen de la situación española en vísperas de la Transición política», Historia del presente, 11: 97-110.

Magallón-Rosa, R. (2017): «Datos abiertos y acceso a la información pública en la reconstrucción de la historia digital», Historia y Comunicación Social 22 (2): 297-308. https:/ / doi.org/10.5209/HICS.57845

Magallón-Rosa, R. (2008): «La historia multimedia. La transformación de la memoria evenemencial». Historia Actual Online, 17: 169-174.

NoIRET, S. (2018): «Trabajar con el pasado en internet : la historia pública digital y las narraciones de las redes sociales», Ayer, 110: 111-140.

PONS, A. (2020): «De la historia local a la historia pública: algún defecto y ciertas virtudes», Hispania Nova, 1 extraordinario: 52-80.

PONS, A. (2018): «El pasado fue analógico, el futuro es digital. Nuevas formas de escritura histórica», Ayer, 110: 19-50.

PONS, A. (2006): «La historia maleable. A propósito de internet», Hispania, 66 (222): 109-130. https:/ / doi.org/10.3989/hispania.2006.v66.i222.4

Rodríguez Mateos, D.; Hernández-Pérez, T. (2015) «Televisión social en series de ficción y nuevos roles del documentalista audiovisual: el caso de "El Ministerio del Tiempo"», Index.comunicación: Revista científica en el ámbito de la Comunicación Aplicada, 5: 95-120.

Zugasti, R. (2005): «La legitimidad franquista de la Monarquía de Juan Carlos I: un ejercicio de amnesia periodística durante la transición española», Comunicación y sociedad, 18 (2): 141-168. 
